
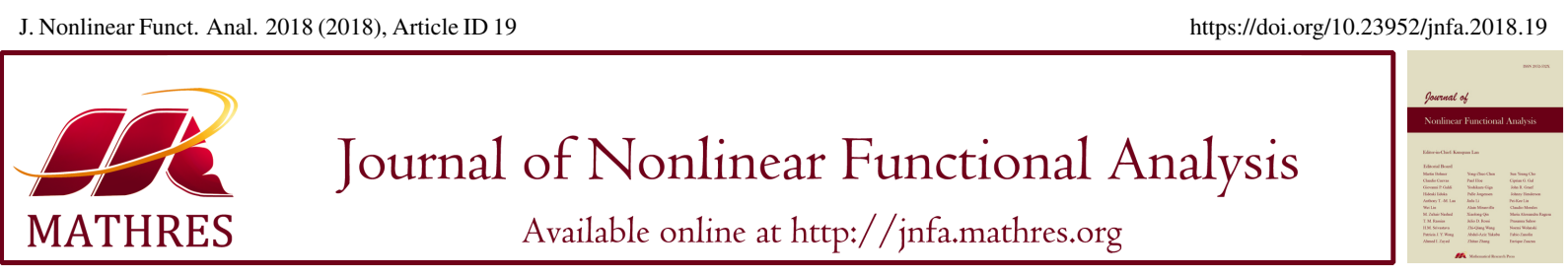

\title{
EXISTENCE OF NONTRIVIAL SOLUTIONS FOR KIRCHHOFF TYPE FRACTIONAL DIFFERENTIAL EQUATIONS WITH LIOUVILLE-WEYL FRACTIONAL DERIVATIVES
}

\author{
ELHAM TAYYEBI, NEMAT NYAMORADI* \\ Department of Mathematics, Faculty of Sciences, Razi University, 67149 Kermanshah, Iran
}

\begin{abstract}
In this paper, we study the existence of nontrivial solutions for Kirchhoff type fractional differential equations. By using the symmetric Mountain Pass Theorem, Morse theory in combination with local linking arguments and the Clark's Theorem, we give some new criteria to guarantee that the Kirchhoff type fractional differential problems have nontrivial solutions. Keywords. Fractional differential equation; Liouville-Weyl fractional derivative; Nontrivial solution; Clark's Theorem; Mountain pass theorem, Morse theory.
\end{abstract}

2010 Mathematics Subject Classification. 34C37, 35A15, 35B38.

\section{INTRODUCTION}

The aim of this paper is to establish the existence of nontrivial solutions for the following Kirchhoff type fractional differential problem:

$$
\left\{\begin{array}{l}
\left(a+\lambda\left(\int_{\mathbb{R}}\left(\left|{ }_{-\infty} D_{t}^{\alpha} u(t)\right|^{2}+b(t)|u(t)|^{2}\right) d t\right)^{\tau}\right) \\
\cdot\left({ }_{t} D_{\infty}^{\alpha}\left({ }_{-\infty} D_{t}^{\alpha} u(t)\right)+b(t) u(t)\right)=f(t, u(t)), \quad t \in \mathbb{R}, \\
u \in H^{\alpha}(\mathbb{R}),
\end{array}\right.
$$

where $\alpha \in(1 / 2,1),{ }_{t} D_{\infty}^{\alpha}$ and ${ }_{-\infty} D_{t}^{\alpha}$ are the right and left inverse operators of the corresponding LiouvilleWeyl fractional derivatives of order $\alpha$ respectively, $H^{\alpha}(\mathbb{R})$ is the classical fractional Sobolev space, $u \in$ $\mathbb{R}$, constants $a>0, \lambda>0, \tau \geq 0, f \in C(\mathbb{R} \times \mathbb{R}, \mathbb{R})$ and $b: \mathbb{R} \rightarrow \mathbb{R}$. Under more relaxed assumptions on $b(t)$ and $f(t, u)$, we first prove the existence of a nontrivial solution for problem (1.1) by using symmetric Mountain Pass Theorem. Then we prove the existence of at least two nontrivial solutions for problem (1.1) by using Morse theory in combination with local linking arguments. Finally, by using the Clark's Theorem, the existence results of at least $k$ distinct pairs of solutions are obtained. Some recent results from the literature are extended.

\footnotetext{
${ }^{*}$ Corresponding author.
}

E-mail addresses: nyamoradi@razi.ac.ir, neamat80@yahoo.com (N. Nyamoradi).

Received January 7, 2018; Accepted May 7, 2018. 
Fractional differential equations have been receiving great interest recently. This is due to both the intensive development of the theory of fractional calculus itself and the applications of such constructions in various scientific fields such as physics, chemistry, biology, economics, control theory, signal and image processing, biophysics, blood flow phenomena, aerodynamics, fitting of experimental data, etc., see $[1,2,3,4,5,6,7]$ and the references therein. In recent years, there has been a growing interest in the study of fractional differential equations with Liouville-Weyl fractional derivatives, for more details and examples, we refer the reader to a series of papers $[8,9,10,11,12,13]$ and the references cited therein.

Recently, Chen and Zhu [14], studied the existence of positive solutions to $p$-Kirchhoff-type problem

$$
\left\{\begin{array}{l}
\left(a+\lambda\left(\int_{\mathbb{R}^{N}}\left(|\nabla u|^{p}+b|u|^{p}\right) d x\right)^{\tau}\right)\left(-\Delta_{p} u+b|u|^{p-2} u\right)=|u|^{m-2} u+\mu|u|^{q-2} u, \quad x \in \mathbb{R}^{N}, \\
u \in W^{1, p}\left(\mathbb{R}^{N}\right), u(x)>0,
\end{array}\right.
$$

where $a, b>0, \lambda, \tau \geq 0 \mu \in \mathbb{R}, 1<p<N, p<q<m<p^{*}=\frac{p N}{N-p}$. They show the existence result by using the Nehari manifold method. Also, many authors are interested in $p$-Kirchhoff type problems, see, for instance, $[15,16,17,18,19,20,21]$ and references therein.

In this paper, motivated by the above papers, we attempt to apply the Mountain Pass Theorem, Morse theory in combination with local linking and Clark's Theorem to study the existence and multiplicity of solutions to problem (1.1).

In view of our problem, we assume that $b(t)$ and $f(t, u)$ satisfy the following hypotheses:

(B) $b: \mathbb{R} \rightarrow(0,+\infty)$ is continuous such that

(B1) $b_{1}=\inf _{t \in \mathbb{R}} b(t)>0$,

(B2) There exists $r_{0}>0$ such that, for any $M>0$

$$
m\left(\left\{t \in\left(y-r_{0}, y+r_{0}\right) / b(t) \leq M\right\}\right) \rightarrow 0 \text { as }|y| \rightarrow \infty .
$$

(f1) $f \in C(\mathbb{R} \times \mathbb{R}, \mathbb{R})$ and $|f(t, u)| \leq C\left(1+|u|^{p-1}\right)$ for some $2<p<\infty$, where $C$ is a positive constant.

(f2) $f(t, u)=o(|u|)$ as $|u| \rightarrow 0$ uniformly in $t \in \mathbb{R}$.

(f3) There exist $\mu>2(\tau+1)$ and $r>0$ such that

$$
\inf _{t \in \mathbb{R},|u|=r} F(t, u)>0
$$

and

$$
\mu F(t, u) \leq f(t, u) u \text { for all } \mathrm{t} \in \mathbb{R} \text { and }|u| \geq r,
$$

where $F(t, u)=\int_{0}^{u} f(t, s) d s$.

(f4) $f \in C(\mathbb{R} \times \mathbb{R}, \mathbb{R})$, and there exist $1<p_{1}<p_{2}<\cdots<p_{m}<2$ and $c_{i}(t) \in L^{\frac{2}{2-p_{i}}}\left(\mathbb{R}, \mathbb{R}^{+}\right)$such that

$$
|f(t, u)| \leq \sum_{i=1}^{m} p_{i} c_{i}(t)|u|^{p_{i}-1}, \forall(t, u) \in \mathbb{R} \times \mathbb{R} .
$$

(f5) There exist $m_{1}>0,0<m_{2}<\frac{a \lambda_{1}}{2}\left(\lambda_{1}\right.$ is defined in Theorem 2.7), $1<\gamma<2$ and small constants $0<r<r_{0}$ such that

$$
m_{1}|u|^{\gamma}<F(t, u) \leq m_{2}|u|^{2}, r \leq|u| \leq r_{0}, \text { a.e. } t \in \mathbb{R} .
$$

(f6) $F(t,-u)=F(t, u), \forall(t, u) \in \mathbb{R} \times \mathbb{R}$.

Our main results read as follows.

Theorem 1.1. Assume that the conditions (B) and (f1)-(f3) hold. Then problem (1.1) has a nontrivial solution. 
Theorem 1.2. Assume that the conditions (B), (f4) and (f5) hold, then problem (1.1) has at least two nontrivial solutions.

Theorem 1.3. Assume that the conditions (B) and (f4)-(f6) hold, then problem (1.1) has at least $k$ distinct nontrivial solutions.

In Section 2, we give preliminary facts and provide some basic properties which are needed later. In Section 3, we prove our main results (Theorems 1.1, 1.2 and 1.3).

\section{PRELIMINARIES}

In this section, we present some basic concepts and lemmas that we need in the sequel, and we refer the reader to $[1,5,8,22,23]$ for basic fractional calculus.

Definition 2.1. [1] The left Liouville-Weyl fractional derivatives of order $0<\alpha<1$ on the whole axis $\mathbb{R}$ is defined by

$$
{ }_{-\infty} D_{x}^{\alpha} u(x)=\frac{\alpha}{\Gamma(1-\alpha)} \int_{0}^{\infty} \frac{u(x)-u(x-\xi)}{\xi^{\alpha+1}} d \xi .
$$

We first define the Fourier transform. Let $u(x)$ be defined on $(-\infty, \infty)$. Then the Fourier transform $\widehat{u}(w)$ of $u(x)$ is defined by

$$
\widehat{u}(w)=\int_{-\infty}^{\infty} e^{-i x \cdot w} u(x) d x .
$$

For $0<\alpha<1$, we give the relationship between classical fractional Sobolev space $H^{\alpha}(\mathbb{R})$ and $I_{-\infty}^{\alpha}(\mathbb{R})$, where the fractional Sobolev space $H^{\alpha}(\mathbb{R})$ is defined by

$$
H^{\alpha}(\mathbb{R})=\overline{C_{0}^{\infty}(\mathbb{R})}\left\|^{\|}\right\|_{\alpha}
$$

with the norm

$$
\|u\|_{\alpha}=\left(\|u\|_{L^{2}}^{2}+|u|_{\alpha}^{2}\right)^{\frac{1}{2}}
$$

and semi-norm

$$
|u|_{\alpha}=\left\||w|^{\alpha} \widehat{u}\right\|_{L^{2}}
$$

Also, we can define

$$
H^{\alpha}(\mathbb{R})=\left\{u \in L^{2}(\mathbb{R}):|w|^{\alpha} \widehat{u} \in L^{2}(\mathbb{R})\right\} .
$$

In what follows, we introduce the fractional space in which we will construct the variational framework of problem (1.1). Let

$$
X^{\alpha}=\left\{u \in H^{\alpha}(\mathbb{R}): \int_{\mathbb{R}}\left(\left|{ }_{-\infty} D_{t}^{\alpha} u(t)\right|^{2}+b(t)|u(t)|^{2}\right) d t<\infty\right\} .
$$

Then $X^{\alpha}$ is a reflexive and separable Hilbert space with the inner product

$$
\langle u, v\rangle_{X^{\alpha}}=\int_{\mathbb{R}}\left({ }_{-\infty} D_{t}^{\alpha} u(t) \cdot{ }_{-\infty} D_{t}^{\alpha} v(t)+b(t) u(t) v(t)\right) d t,
$$

and the corresponding norm

$$
\|u\|_{X^{\alpha}}^{2}=\langle u, u\rangle_{X^{\alpha}} .
$$

Similar to proofs of Lemma 2.1, Lemma 2.2 and Theorem 2.1 in [9], we can get the following lemmas. 
Lemma 2.2. [9] Suppose b satisfies (B). Then the space $X^{\alpha}$ is continuously embedded in $H^{\alpha}(\mathbb{R})$.

Lemma 2.3. ([9]) Suppose b satisfies (B). Then the imbedding of $X^{\alpha}$ in $L^{2}(\mathbb{R})$ is continuous and compact.

Lemma 2.4. ([9]) If $\alpha>\frac{1}{2}$, then $H^{\alpha}(\mathbb{R}) \subset C(\mathbb{R})$ and there is a constant $c=c_{\alpha}$ such that

$$
\sup _{x \in \mathbb{R}}|u(x)| \leq c\|u\|_{\alpha},
$$

From Lemma 2.4, we find that there exists a constant $c_{\alpha}>0$ such that $\|u\|_{\infty} \leq c_{\alpha}\|u\|_{X^{\alpha}}$.

Remark 2.5. From Lemma 2.4, we know that if $u \in H^{\alpha}(\mathbb{R})$ with $\frac{1}{2}<\alpha<1$, then $u \in L^{p}(\mathbb{R})$ for all $p \in[2, \infty)$. The reason is

$$
\int_{\mathbb{R}}|u(x)|^{p} d x \leq\|u\|_{\infty}^{p-2} \cdot\|u\|_{L^{2}}^{2} .
$$

Remark 2.6. From Remark 2.5 and Lemma 2.3 , it is easy to verify that the imbedding of $X^{\alpha}$ in $L^{p}(\mathbb{R})$ is also compact for $p \in(2, \infty)$. Hence, for all $2 \leq p<\infty$, the imbedding of $X^{\alpha}$ in $L^{p}(\mathbb{R})$ is continuous and compact, which together with Lemma 2.4 implies that, for all $p \in[2, \infty)$, there exists $c_{p}>0$ such that

$$
\|u\|_{L^{p}(\mathbb{R})} \leq C_{p}\|u\|_{X^{\alpha}} .
$$

Now, we consider the eigenvalue problem

$$
\left\{\begin{array}{l}
{ }_{t} D_{\infty}^{\alpha}\left({ }_{-\infty} D_{t}^{\alpha} u(t)\right)+b(t) u(t)=\lambda u, \quad t \in \mathbb{R}, \\
u \in H^{\alpha}(\mathbb{R}) .
\end{array}\right.
$$

Theorem 2.7. [11, Theorem 1] Suppose that (B) holds. Then each eigenvalue of (2.5) is real and if repeatting each eigenvalue according to its multiplicity, we have $0<\lambda_{1} \leq \lambda_{2} \leq \lambda_{3} \leq \ldots$ and $\lambda_{k} \rightarrow \infty$ as $k \rightarrow \infty$. And $\lambda_{1}$ can be characterized as

$$
\lambda_{1}=\inf _{u \in X^{\alpha} \backslash\{0\}} \frac{\int_{\mathbb{R}}\left(\left|{ }_{-\infty} D_{t}^{\alpha} u(t)\right|^{2}+b(t)|u(t)|^{2}\right) d t}{\int_{\mathbb{R}}|u(t)|^{2} d t} .
$$

Furthermore, there exists an orthogonal basis $\left\{w_{k}\right\}_{k=1}^{\infty}$ of $X^{\alpha}$, where $w_{k} \in X^{\alpha}$ is an eigenfunction corresponding to $\lambda_{k}$ for $k=1,2, \ldots$.

Definition 2.8. We say that $\varphi$ satisfies the Palais-Smale condition (PS)-condition if for any sequence $\left\{u_{n}\right\} \subset X$ which $\varphi\left(u_{n}\right)$ is bounded and $\varphi^{\prime}\left(u_{n}\right) \rightarrow 0$ as $n \rightarrow \infty$ possesses a convergent subsequence.

Definition 2.9. Let $\varphi: X \rightarrow \mathbb{R}$ be differentiable and $c \in \mathbb{R}$. We say that $\varphi$ satisfies the (PS) $)_{\mathrm{c}}$-condition if the existence of a sequence $\left\{u_{n}\right\} \subset X$ such that

$$
\varphi\left(u_{n}\right) \rightarrow c, \varphi^{\prime}\left(u_{n}\right) \rightarrow 0
$$

as $n \rightarrow \infty$, implies that $c$ is a critical value of $\varphi$.

Remark 2.10. It is clear that the (PS)-condition implies the $(\mathrm{PS})_{\mathrm{c}}$-condition for each $c \in \mathbb{R}$.

Definition 2.11. Let $X$ be a real Banach space and $\varphi \in C^{1}(X, \mathbb{R}) . K=\left\{u \in X: \varphi^{\prime}(u)=0\right\}$, then the qth critical group of $\varphi$ at an isolated critical point $u \in K$ with $\varphi(u)=c$ is defined by

$$
C_{q}(\varphi, u):=H_{q}\left(\varphi^{c} \cap U, \varphi^{c} \cap U \backslash\{u\}\right), q \in \mathbb{N} \cup\{0\},
$$

where $\varphi^{c}=\{u \in X: \varphi(u) \leq c\}$, where $U$ is a neighborhood of $u$, containing the unique critical point, $H_{q}(\cdot, \cdot)$ stands for the $q$ th singular relative homology group with integer coefficients. 
We say that $u \in X$ is a homological nontrivial critical point of $\varphi$ if at least one of its critical groups is nontrivial.

Now, we present the following Theorems that will be used later.

Theorem 2.12. [24] Let $X$ be a Banach space and $\varphi \in C^{1}(X, \mathbb{R})$. Assume that there exist $u_{0} \in X, u_{1} \in X$ and a bounded open neighborhood $\Omega$ of $u_{0}$ such that $u_{1} \in X \backslash \Omega$ and

$$
\inf _{\partial \Omega} \varphi>\max \left(\varphi\left(u_{0}\right), \varphi\left(u_{1}\right)\right) .
$$

Let $\Gamma=\left\{g \in C([0,1], X): g(0)=u_{0}, g(1)=u_{1}\right\}$ and $c=\inf _{g \in \Gamma} \max _{s \in[0,1]} \varphi(g(s))$. If $\varphi$ satisfies the $(P S)_{c^{-}}$ condition, then $c$ is a critical value of $\varphi$ and $c>\max \left(\varphi\left(u_{0}\right), \varphi\left(u_{1}\right)\right)$.

Theorem 2.13. [25] Assume that $\varphi$ has a critical point $u=0$ with $\varphi(0)=0$. Suppose that $\varphi$ has a local linking at 0 with respect to $X=V \oplus W, K=\operatorname{dim} V<\infty$, that is, there exists $\rho>0$ small such that

$$
\left\{\begin{array}{l}
\varphi(u) \leq 0, u \in V,\|u\| \leq \rho ; \\
\varphi(u)>0, u \in W, 0<\|u\| \leq \rho .
\end{array}\right.
$$

Then $C_{k}(\varphi, 0) \supsetneqq 0$. Hence 0 is a homological nontrivial critical point of $\varphi$.

Theorem 2.14. [25] Let $X$ be a real Banach space and let $\varphi \in C^{1}(X, \mathbb{R})$ be bounded from below and satisfy the (PS)-condition. If $\varphi$ has a critical point that is homological nontrivial and is not a minimizer of $\varphi$, then $\varphi$ has at least three critical points.

Theorem 2.15. [26] Let $\varphi \in C^{1}(X, \mathbb{R})$ with $\varphi$ even, satisfying (PS)-condition and bounded from below. Assume that $\varphi(0)=0$. There is a set $K \subset X$ such that $K$ is homeomorphic to $S^{j-1}$ by an odd map, where $S^{j-1}$ denotes the $j$-dimensional unit sphere, and $\sup _{K} \varphi<0$. Then $\varphi$ has at least $j$ distinct pairs of critical points.

A weak solution of problem (1.1) is a critical point of the following functional

$$
\begin{aligned}
\varphi(u)= & \frac{a}{2} \int_{\mathbb{R}}\left(\left.\left.\right|_{-\infty} D_{t}^{\alpha} u(t)\right|^{2}+b(t)|u(t)|^{2}\right) d t+\frac{\lambda}{2(\tau+1)}\left(\int_{\mathbb{R}}\left(\left|-_{-\infty} D_{t}^{\alpha} u(t)\right|^{2}+b(t)|u(t)|^{2}\right) d t\right)^{\tau+1} \\
& -\int_{\mathbb{R}} F(t, u(t)) d t
\end{aligned}
$$

for all $u \in X^{\alpha}$.

Lemma 2.16. Assume that (B), (f1) and (f2) ( or (B) and (f4)) hold. Then $\varphi \in C^{1}\left(X^{\alpha}, \mathbb{R}\right)$ and

$$
\begin{aligned}
\left\langle\varphi^{\prime}(u), v\right\rangle= & \left(a+\lambda\left(\int_{\mathbb{R}}\left(\left.\left.\right|_{-\infty} D_{t}^{\alpha} u(t)\right|^{2}+b(t)|u(t)|^{2}\right) d t\right)^{\tau}\right) \\
& \cdot \int_{\mathbb{R}}\left({ }_{-\infty} D_{t}^{\alpha} u(t)_{-\infty} D_{t}^{\alpha} v(t)+b(t) u(t) v(t)\right) d t-\int_{\mathbb{R}} f(t, u(t)) v(t) d t,
\end{aligned}
$$

for all $u, v \in X^{\alpha}$.

Proof. First we suppose that the conditions (B), (f1) and (f2) hold. Set $\psi(u)=\int_{\mathbb{R}} F(t, u(t)) d t$. According to (2.7), it suffices to show that $\psi \in C^{1}\left(X^{\alpha}, \mathbb{R}\right)$,

$$
\left\langle\psi^{\prime}(u), v\right\rangle=\int_{\mathbb{R}} f(t, u(t)) v(t) d t, \quad \forall u, v \in X^{\alpha} .
$$


To prove $\varphi \in C^{1}\left(X^{\alpha}, \mathbb{R}\right)$ and (2.8), First, we show the existence of the Gateaux derivative of $\psi$. For $\varepsilon>0$, by (f1) and (f2), we find that there is $c(\varepsilon)>0$ such that

$$
|f(t, u)| \leq \varepsilon|u|+c(\varepsilon)|u|^{p-1}
$$

and

$$
|F(t, u)| \leq \frac{\varepsilon}{2}|u|^{2}+\frac{1}{p} c(\varepsilon)|u|^{p}
$$

for all $(t, u) \in \mathbb{R} \times \mathbb{R}$. For any $u, v \in X^{\alpha}$ and $0<|\mu|<1$, by the mean value theorem and (2.9), we find that there exists $0<\theta<1$ such that

$$
f(t, u(t)+\theta \mu v(t))=\frac{F(t, u(t)+\mu v(t))-F(t, u(t))}{\mu v(t)},
$$

and

$$
\begin{aligned}
& \left|\frac{F(t, u(t)+\mu v(t))-F(t, u(t))}{\mu}\right| \\
= & |f(t, u(t)+\theta \mu v(t))| \cdot|v(t)| \\
\leq & \left(\varepsilon|u(t)+\theta \mu v(t)|+c(\varepsilon)|u(t)+\theta \mu v(t)|^{p-1}\right) \cdot|v(t)| \\
\leq & \varepsilon|u(t)| \cdot|v(t)|+\varepsilon|v(t)|^{2}+c(\varepsilon)\left(2^{p-1}\left(|u(t)|^{p-1}+|v(t)|^{p-1}\right)\right) \cdot|v(t)| \\
= & \varepsilon|u(t)| \cdot|v(t)|+\varepsilon|v(t)|^{2}+2^{p-1} c(\varepsilon)\left(|u(t)|^{p-1} \cdot|v(t)|+|v(t)|^{p}\right) .
\end{aligned}
$$

The Hölder inequality implies that

$$
g(t):=\varepsilon|u(t)| \cdot|v(t)|+\varepsilon|v(t)|^{2}+2^{p-1} c(\varepsilon)\left(|u(t)|^{p-1} \cdot|v(t)|+|v(t)|^{p}\right) \in L^{1}(\mathbb{R}) .
$$

Then, by the Lebesgue's Dominated Convergence Theorem, we have

$$
\begin{aligned}
\left\langle\psi^{\prime}(u), v\right\rangle & =\lim _{\mu \rightarrow 0^{+}} \frac{\psi(u+\mu v)-\psi(u)}{\mu} \\
& =\lim _{\mu \rightarrow 0^{+}} \int_{\mathbb{R}} \frac{F(t, u(t)+\mu v(t))-F(t, u(t))}{\mu} d t \\
& =\lim _{\mu \rightarrow 0^{+}} \int_{\mathbb{R}} f(t, u(t)+\theta \mu v(t)) v(t) d t=\int_{\mathbb{R}} f(t, u(t)) v(t) d t .
\end{aligned}
$$

Hence, from (2.7), one can get

$$
\begin{aligned}
& \frac{\varphi(u+\mu v)-\varphi(u)}{\mu} \\
= & \frac{a}{2} \int_{\mathbb{R}}\left(\left.\left.\mu\right|_{-\infty} D_{t}^{\alpha} v(t)\right|^{2}+2_{-\infty} D_{t}^{\alpha} u(t)_{-\infty} D_{t}^{\alpha} v(t)+b(t)\left(\mu|v(t)|^{2}+2 u(t) v(t)\right)\right) d t \\
& +\frac{\lambda}{2 \mu(\tau+1)}\left[\left(\int _ { \mathbb { R } } \left(\left.\left.\right|_{-\infty} D_{t}^{\alpha} u(t)\right|^{2}+\left.\left.\mu^{2}\right|_{-\infty} D_{t}^{\alpha} v(t)\right|^{2}+2 \mu_{-\infty} D_{t}^{\alpha} u(t)_{-\infty} D_{t}^{\alpha} v(t)\right.\right.\right. \\
& \left.\left.+b(t)\left(|u(t)|^{2}+\mu^{2}|v(t)|^{2}+2 \mu u(t) v(t)\right)\right) d t\right)^{\tau+1} \\
& \left.-\left(\int_{\mathbb{R}}\left(\left.||_{-\infty} D_{t}^{\alpha} u(t)\right|^{2}+b(t)|u(t)|^{2}\right) d t\right)^{\tau+1}\right] \\
& -\int_{\mathbb{R}} \frac{F(t, u(t)+\mu v(t))-F(t, u(t))}{\mu} d t
\end{aligned}
$$


Letting $\mu \rightarrow 0^{+}$, we obtain (2.8).

Next, we show that $\psi^{\prime}(\cdot): X^{\alpha} \rightarrow\left(X^{\alpha}\right)^{*}$ is continuous. Assume that $u_{n} \rightarrow u$ in $X^{\alpha}$. By (2.4) and Remark 2.6, one has $u_{n} \rightarrow u$ in $L^{p}(\mathbb{R})$. Note that

$$
\begin{aligned}
\left\|\psi^{\prime}\left(u_{n}\right)-\psi^{\prime}(u)\right\|_{X^{\alpha}} & =\sup _{\|v\| \leq 1}\left|\int_{\mathbb{R}}\left(f\left(t, u_{n}\right)-f(t, u)\right) v d t\right| \\
& \leq \sup _{\|v\| \leq 1} \int_{\mathbb{R}}\left|f\left(t, u_{n}\right)-f(t, u)\right| \cdot|v| d t
\end{aligned}
$$

and

$$
|f(t, u)| \leq \varepsilon|u|+c(\varepsilon)|u|^{p-1}=\varepsilon|u|^{\frac{2}{2}}+c(\varepsilon)|u|^{\frac{p}{p^{\prime}}},
$$

where $p^{\prime}=\frac{p}{p-1}$. By the Hölder inequality, Theorem A.4 in [27], we have

$$
\begin{gathered}
\left|f\left(t, u_{n}\right)-f(t, u)\right| \rightarrow 0, \\
\sup _{\|v\| \leq 1} \int_{\mathbb{R}}\left|f\left(t, u_{n}\right)-f(t, u)\right| \cdot|v| d t \rightarrow 0, \text { as } n \rightarrow \infty .
\end{gathered}
$$

Hence

$$
\left\|\psi^{\prime}\left(u_{n}\right)-\psi^{\prime}(u)\right\|_{X^{\alpha}} \rightarrow 0
$$

This shows that $\psi^{\prime}$ is continuous. This completes the proof. The proof of this lemma with the conditions (B) and (f4) is similar and we omit it here.

Lemma 2.17. Suppose that (f1) and (f2) hold. Then $\psi(u)=\int_{\mathbb{R}} F(t, u(t)) d t$ is weakly continuous on $X^{\alpha}$.

Proof. Let $\left\{u_{n}\right\} \subset X^{\alpha}$ converge weakly to $\mathrm{u}$ in $X^{\alpha}$. Then $\left\{u_{n}\right\}$ is bounded in $X^{\alpha}$. Note that $X^{\alpha}$ is a reflexive Banach space. Passing to a subsequence (for simplicity denoted again by $\left\{u_{n}\right\}$ ) if necessary, by Remark 2.6, we may assume that

$$
\begin{cases}u_{n} \rightarrow u, & \text { weakly in } X^{\alpha}, \\ u_{n} \rightarrow u, & \text { strongly in } L^{p}(\mathbb{R}), \quad 2 \leq p<\infty\end{cases}
$$

It follows that

$$
\frac{d}{d s}\left(F\left(t, u+s\left(u_{n}-u\right)\right)\right)=\left(u_{n}-u\right) f\left(t, u+s\left(u_{n}-u\right)\right)
$$

This implies that

$$
F\left(t, u_{n}\right)-F(t, u)=\int_{0}^{1} f\left(t, u+s\left(u_{n}-u\right)\right) \cdot\left(u_{n}-u\right) d s .
$$


From (2.9), (2.16) and Hölder inequality, we can get

$$
\begin{aligned}
& \left|\psi\left(u_{n}\right)-\psi(u)\right| \\
= & \left|\int_{\mathbb{R}}\left(F\left(t, u_{n}\right)-F(t, u)\right) d t\right| \\
= & \left|\int_{\mathbb{R}}\left(\int_{0}^{1} f\left(t, u+s\left(u_{n}-u\right)\right) \cdot\left(u_{n}-u\right) d s\right) d t\right| \\
\leq & \int_{\mathbb{R}} \int_{0}^{1}\left|f\left(t, u+s\left(u_{n}-u\right)\right)\right| \cdot\left|u_{n}-u\right| d s d t \\
\leq & \int_{\mathbb{R}} \int_{0}^{1}\left(\varepsilon\left|u+s\left(u_{n}-u\right)\right|+c(\varepsilon)\left|u+s\left(u_{n}-u\right)\right|^{p-1}\right) \cdot\left|u_{n}-u\right| d s d t \\
\leq & \int_{\mathbb{R}} \int_{0}^{1}\left(\varepsilon\left(|u|+s\left|u_{n}-u\right|\right)+c(\varepsilon, p)\left(|u|^{p-1}+s^{p-1}\left|u_{n}-u\right|^{p-1}\right)\right) \cdot\left|u_{n}-u\right| d s d t \\
\leq & \int_{\mathbb{R}}\left(\varepsilon\left(|u|+\frac{1}{2}\left|u_{n}-u\right|\right)+c(\varepsilon, p)\left(|u|^{p-1}+\left|u_{n}-u\right|^{p-1}\right)\right) \cdot\left|u_{n}-u\right| d t \\
= & \varepsilon \int_{\mathbb{R}}\left(|u|+\frac{1}{2}\left|u_{n}-u\right|\right) \cdot\left|u_{n}-u\right| d t+c(\varepsilon, p) \int_{\mathbb{R}}\left(|u|^{p-1}+\left|u_{n}-u\right|^{p-1}\right) \\
& \cdot\left|u_{n}-u\right| d t \\
\leq & \varepsilon\left(\|u\|_{2}+\frac{1}{2}\left\|u_{n}-u\right\|_{2}\right)\left\|u_{n}-u\right\|_{2}+c_{1} c(\varepsilon, p)\left\|u_{n}-u\right\|_{p},
\end{aligned}
$$

where $c(\varepsilon, p)$ is a positive constant and $c_{1}$ is independent of $\varepsilon$ and $\mathrm{n}$. Since by (2.15),

$$
\left\|u_{n}-u\right\|_{p} \rightarrow 0,\left\|u_{n}-u\right\|_{2} \rightarrow 0
$$

as $n \rightarrow \infty$, one has

$$
\left\|\psi\left(u_{n}\right)-\psi(u)\right\|_{X^{\alpha}} \rightarrow 0 \text { as } n \rightarrow \infty .
$$

This shows that $\psi$ is weakly continuous on $X^{\alpha}$. This completes the proof.

\section{PRoOF OF THE MAIN RESUlts}

In this section, we will prove Theorem 1.1, Theorem 1.2 and Theorem 1.3. To complete the proof, we need the following lemmas.

Lemma 3.1. If $(B)$ and (f4) hold, then $\varphi$ is bounded from below and satisfies the (PS)-condition.

Proof. By (f4), we have

$$
|F(t, u)| \leq \sum_{i=1}^{m} c_{i}(t)|u|^{p_{i}}, \quad \forall(t, u) \in \mathbb{R} \times \mathbb{R}
$$

By (B1) and the Hölder inequality, we get 


$$
\begin{aligned}
\sum_{i=1}^{m} \int_{\mathbb{R}} c_{i}(t)|u(t)|^{p_{i}} d t & \leq \sum_{i=1}^{m} \int_{\mathbb{R}} c_{i}(t) \frac{b(t)^{\frac{p_{i}}{2}}}{b_{1}^{\frac{p_{i}}{2}}}|u(t)|^{p_{i}} d t \\
& =\sum_{i=1}^{m} b_{1}^{-\frac{p_{i}}{2}} \int_{\mathbb{R}}\left|c_{i}(t)\right| b(t)^{\frac{p_{i}}{2}}|u(t)|^{p_{i}} d t \\
& \leq \sum_{i=1}^{m} b_{1}^{-\frac{p_{i}}{2}}\left(\int_{\mathbb{R}}\left|c_{i}(t)\right|^{\frac{2}{2-p_{i}}} d t\right)^{\frac{2-p_{i}}{2}}\left(\int_{\mathbb{R}}\left(b(t)^{\frac{p_{i}}{2}}|u(t)|^{p_{i}}\right)^{\frac{2}{p_{i}}} d t\right)^{\frac{p_{i}}{2}} \\
& =\sum_{i=1}^{m} b_{1}^{-\frac{p_{i}}{2}}\left\|c_{i}\right\|_{\frac{2}{2-p_{i}}}\left(\int_{\mathbb{R}} b(t)|u(t)|^{2} d t\right)^{\frac{p_{i}}{2}} \\
& \leq \sum_{i=1}^{m} b_{1}^{-\frac{p_{i}}{2}}\left\|c_{i}\right\|_{\frac{2}{2-p_{i}}}\|u\|_{X^{\alpha}}^{p_{i}} .
\end{aligned}
$$

In view of (2.7), (3.1), (3.2) and Hölder inequality, one can get

$$
\begin{aligned}
& \varphi(u) \\
= & \frac{a}{2} \int_{\mathbb{R}}\left(\left.||_{-\infty} D_{t}^{\alpha} u(t)\right|^{2}+b(t)|u(t)|^{2}\right) d t+\frac{\lambda}{2(\tau+1)}\left(\int_{\mathbb{R}}\left(\left|{ }_{-\infty} D_{t}^{\alpha} u(t)\right|^{2}+b(t)|u(t)|^{2}\right) d t\right)^{\tau+1} \\
& -\int_{\mathbb{R}} F(t, u(t)) d t \\
= & \frac{a}{2}\|u\|_{X^{\alpha}}^{2}+\frac{\lambda}{2(\tau+1)}\|u\|_{X^{\alpha}}^{2(\tau+1)}-\int_{\mathbb{R}} F(t, u(t)) d t \\
\geq & \frac{a}{2}\|u\|_{X^{\alpha}}^{2}-\int_{\mathbb{R}} F(t, u(t)) d t \\
\geq & \frac{a}{2}\|u\|_{X^{\alpha}}^{2}-\sum_{i=1}^{m} \int_{\mathbb{R}} c_{i}(t)|u(t)|^{p_{i}} d t \\
\geq & \frac{a}{2}\|u\|_{X^{\alpha}}^{2}-\sum_{i=1}^{m} b_{1}^{-\frac{p_{i}}{2}}\left\|c_{i}\right\|_{\frac{2}{2-p_{i}}}\|u\|_{X^{\alpha}}^{p_{i}}
\end{aligned}
$$

which implies that $\varphi(u) \rightarrow+\infty$, as $\|u\|_{X^{\alpha}} \rightarrow \infty$, because $a>0, \lambda>0, \tau \geq 0$ and $1<p_{i}<2(i=$ $1,2, \ldots, m)$. So, $\varphi$ is bounded from below.

Next, we prove that $\varphi$ satisfies the (PS)-condition. To this end, we assume that $\left\{u_{n}\right\}$ is a $(P S)$-sequence of $\varphi$ such that $\left\{\varphi\left(u_{n}\right)\right\}$ is bounded and $\varphi^{\prime}\left(u_{n}\right) \rightarrow 0$, as $n \rightarrow \infty$. Then, it follows from (3.3) that there exists a constant $c>0$ such that

$$
c \geq \varphi\left(u_{n}\right) \geq \frac{a}{2}\left\|u_{n}\right\|_{X^{\alpha}}^{2}-\sum_{i=1}^{m} b_{1}^{-\frac{p_{i}}{2}}\left\|c_{i}\right\|_{\frac{2}{2-p_{i}}}\left\|u_{n}\right\|_{X^{\alpha}}^{p_{i}}
$$

Consequently, it follows from (2.4) that there exists a constant $c_{4}>0$ such that

$$
\left\|u_{n}\right\|_{2} \leq c_{4}\left\|u_{n}\right\|_{X^{\alpha}} \leq c, n \in \mathbb{N} .
$$

This implies that $\left\{u_{n}\right\}$ is bounded. Since $\left\{u_{n}\right\} \subset X^{\alpha}$ is bounded and $X^{\alpha}$ is a reflexive Banach space and so by passing to a subsequence (for simplicity denoted again by $\left\{u_{n}\right\}$ ) if necessary, by Remark 2.6, we 
may assume that

$$
\left\{\begin{array}{l}
u_{n} \rightarrow u, \quad \text { weakly in } X^{\alpha}, \\
u_{n} \rightarrow u, \quad \text { strongly in } L^{p}(\mathbb{R}), \quad 2 \leq p<\infty .
\end{array}\right.
$$

On the other hand, for any given $\varepsilon>0$, by $\left(f_{4}\right)$, we can choose $R_{\varepsilon}>0$ such that

$$
\left(\int_{|t|>R_{\varepsilon}}\left|c_{i}(t)\right|^{\frac{2}{2-p_{i}}} d t\right)^{\frac{2-p_{i}}{2}}<\varepsilon, i=1,2, \ldots, m .
$$

It follows from (3.6) that there exists $n_{0}>0$ such that

$$
\int_{|t| \leq R_{\varepsilon}}\left|u_{n}-u\right|^{2} d t<\varepsilon^{2}, \text { for } n \geq n_{0} .
$$

Thus, by (f4), (3.5), (3.8) and the Hölder inequality, for any $n \geq n_{0}$, one has

$$
\begin{aligned}
& \int_{|t| \leq R_{\varepsilon}}\left|f\left(t, u_{n}\right)-f(t, u)\right|\left|u_{n}-u\right| d t \\
\leq & \left(\int_{|t| \leq R_{\varepsilon}}\left|f\left(t, u_{n}\right)-f(t, u)\right|^{2} d t\right)^{\frac{1}{2}}\left(\int_{|t| \leq R_{\varepsilon}}\left|u_{n}-u\right|^{2} d t\right)^{\frac{1}{2}} \\
\leq & \varepsilon\left(\int_{|t| \leq R_{\varepsilon}}\left|f\left(t, u_{n}\right)-f(t, u)\right|^{2} d t\right)^{\frac{1}{2}} \\
\leq & \varepsilon\left(\int_{|t| \leq R_{\varepsilon}} 2\left(\left|f\left(t, u_{n}\right)\right|^{2}+|f(t, u)|^{2}\right) d t\right)^{\frac{1}{2}} \\
\leq & \varepsilon\left[\int_{|t| \leq R_{\varepsilon}} 2\left(\left(\sum_{i=1}^{m} p_{i} c_{i}(t)\left|u_{n}\right|^{p_{i}-1}\right)^{2}+\left(\sum_{i=1}^{m} p_{i} c_{i}(t)|u|^{p_{i}-1}\right)^{2}\right) d t\right]^{\frac{1}{2}} \\
\leq & 2 \varepsilon\left(\sum_{i=1}^{m} p_{i}^{2}\left\|c_{i}\right\|_{\frac{2}{2-p_{i}}}^{2}\left(\left\|u_{n}\right\|_{2}^{2\left(p_{i}-1\right)}+\|u\|_{2}^{2\left(p_{i}-1\right)}\right)\right)^{\frac{1}{2}} \\
\leq & 2 \varepsilon\left(\sum_{i=1}^{m} p_{i}^{2}\left\|c_{i}\right\|_{\frac{2}{2-p_{i}}}^{2}\left(c^{2\left(p_{i}-1\right)}+\|u\|_{2}^{2\left(p_{i}-1\right)}\right)\right)^{\frac{1}{2}} .
\end{aligned}
$$

Also, for $n \in \mathbb{N}$, (f4), (3.5), (3.7) and Hölder inequality imply that

$$
\begin{aligned}
\int_{|t|>R_{\varepsilon}}\left|f\left(t, u_{n}\right)-f(t, u)\right|\left|u_{n}-u\right| d t & \leq \sum_{i=1}^{m} p_{i} \int_{|t|>R_{\varepsilon}}\left|c_{i}(t)\right|\left(\left|u_{n}\right|^{p_{i}-1}+|u|^{p_{i}-1}\right)\left(\left|u_{n}\right|+|u|\right) d t \\
& \leq 2 \sum_{i=1}^{m} p_{i} \int_{|t|>R_{\varepsilon}}\left|c_{i}(t)\right|\left(\left|u_{n}\right|^{p_{i}}+|u|^{p_{i}}\right) d t \\
& =2 \sum_{i=1}^{m} p_{i}\left(\int_{|t|>R_{\varepsilon}}\left|c_{i}(t)\right|\left|u_{n}\right|^{p_{i}} d t+\int_{|t|>R_{\varepsilon}}\left|c_{i}(t)\right||u|^{p_{i}} d t\right) \\
& \leq 2 \sum_{i=1}^{m} p_{i}\left(\int_{|t|>R_{\varepsilon}}\left|c_{i}(t)\right|^{\frac{2}{2-p_{i}}} d t\right)^{\frac{2-p_{i}}{2}}\left(\left\|u_{n}\right\|_{2}^{p_{i}}+\|u\|_{2}^{p_{i}}\right) \\
& \leq 2 \varepsilon \sum_{i=1}^{m} p_{i}\left(\left\|u_{n}\right\|_{2}^{p_{i}}+\|u\|_{2}^{p_{i}}\right) \\
& \leq 2 \varepsilon \sum_{i=1}^{m} p_{i}\left(c^{p_{i}}+\|u\|_{2}^{p_{i}}\right) .
\end{aligned}
$$


Since $\varepsilon$ is arbitrary, we find that (3.9) and (3.10) imply that

$$
\lim _{n \rightarrow \infty} \int_{\mathbb{R}}\left(f\left(t, u_{n}\right)-f(t, u)\right)\left(u_{n}-u\right) d t=0 .
$$

Then by (2.8) (similar to the proof of Lemma 3.3), one has

$$
\begin{aligned}
& o_{n}(1)=\left\langle\varphi^{\prime}\left(u_{n}\right)-\varphi^{\prime}(u), u_{n}-u\right\rangle \\
& =a \int_{\mathbb{R}}\left(\left.\left.\right|_{-\infty} D_{t}^{\alpha}\left(u_{n}-u\right)\right|^{2}+b(t)\left|u_{n}-u\right|^{2}\right) d t \\
& -\lambda\left[\left(\int_{\mathbb{R}}\left(\left.\left.\right|_{-\infty} D_{t}^{\alpha} u\right|^{2}+b(t)|u|^{2}\right) d t\right)^{\tau}\right. \\
& \cdot \int_{\mathbb{R}}\left({ }_{-\infty} D_{t}^{\alpha} u_{-\infty} D_{t}^{\alpha}\left(u_{n}-u\right)+b(t) u\left(u_{n}-u\right)\right) d t \\
& -\left(\int_{\mathbb{R}}\left(\left.\left.\right|_{-\infty} D_{t}^{\alpha} u_{n}\right|^{2}+b(t)\left|u_{n}\right|^{2}\right) d t\right)^{\tau} \\
& \left.\cdot \int_{\mathbb{R}}\left({ }_{-\infty} D_{t}^{\alpha} u_{n-\infty} D_{t}^{\alpha}\left(u_{n}-u\right)+b(t) u_{n}\left(u_{n}-u\right)\right) d t\right] \\
& -\int_{\mathbb{R}}\left(f\left(t, u_{n}\right)-f(t, u)\right) \cdot\left(u_{n}-u\right) d t \\
& =a\left\|u_{n}-u\right\|_{X^{\alpha}}^{2} \\
& -\lambda\left[\|u\|_{X^{\alpha}}^{2 \tau} \cdot \int_{\mathbb{R}}\left({ }_{-\infty} D_{t}^{\alpha} u{ }_{-\infty} D_{t}^{\alpha}\left(u_{n}-u\right)+b(t) u\left(u_{n}-u\right)\right) d t\right. \\
& \left.-\left\|u_{n}\right\|_{X^{\alpha}}^{2 \tau} \cdot \int_{\mathbb{R}}\left({ }_{-\infty} D_{t}^{\alpha} u_{n-\infty} D_{t}^{\alpha}\left(u_{n}-u\right)+b(t) u_{n}\left(u_{n}-u\right)\right) d t\right] \\
& -\int_{\mathbb{R}}\left(f\left(t, u_{n}\right)-f(t, u)\right) \cdot\left(u_{n}-u\right) d t \\
& =a\left\|u_{n}-u\right\|_{X^{\alpha}}^{2} \\
& -\lambda\left[\|u\|_{X^{\alpha}}^{2 \tau} \cdot \int_{\mathbb{R}}\left({ }_{-\infty} D_{t}^{\alpha} u-_{-\infty} D_{t}^{\alpha}\left(u_{n}-u\right)+b(t) u\left(u_{n}-u\right)\right) d t\right. \\
& \left.-\left\|u_{n}\right\|_{X^{\alpha}}^{2 \tau} \cdot \int_{\mathbb{R}}\left({ }_{-\infty} D_{t}^{\alpha} u_{n-\infty} D_{t}^{\alpha}\left(u_{n}-u\right)+b(t) u_{n}\left(u_{n}-u\right)\right) d t\right] \\
& -\int_{\mathbb{R}}\left(f\left(t, u_{n}\right)-f(t, u)\right) \cdot\left(u_{n}-u\right) d t .
\end{aligned}
$$

Hence, by the boundedness of $\left\{u_{n}\right\}$ and the weak convergence of $\left\{u_{n}\right\}$, we get

$$
\begin{aligned}
& \lambda\left[\|u\|_{X^{\alpha}}^{2 \tau} \cdot \int_{\mathbb{R}}\left({ }_{-\infty} D_{t}^{\alpha} u{ }_{-\infty} D_{t}^{\alpha}\left(u_{n}-u\right)+b(t) u\left(u_{n}-u\right)\right) d t\right. \\
& \left.-\left\|u_{n}\right\|_{X^{\alpha}}^{2 \tau} \cdot \int_{\mathbb{R}}\left({ }_{-\infty} D_{t}^{\alpha} u_{n-\infty} D_{t}^{\alpha}\left(u_{n}-u\right)+b(t) u_{n}\left(u_{n}-u\right)\right) d t\right] \rightarrow 0 \text {, as } n \rightarrow \infty .
\end{aligned}
$$

Thus, by using (3.11), (3.12) and (3.13), we have that $\left\|u_{n}-u\right\|_{X^{\alpha}} \rightarrow 0$. Therefore, $\varphi$ satisfies the (PS)condition. The proof is complete.

Lemma 3.2. If conditions $(B)$ and (f4)-(f5) hold, then there exists $k_{0} \in \mathbb{N}$ such that $C_{k_{0}}(\varphi, 0) \varsubsetneqq 0$.

Proof. By (2.7), one can easily check that functional $\varphi$ satisfies $\varphi(0)=0$. It follows from (f4) and (2.8), we can get $\left\langle\varphi^{\prime}(0), v\right\rangle=0 \forall v \in X^{\alpha}$, so $\varphi^{\prime}(0)=0$. Consequently, the zero function is a critical point of $\varphi$. So we only need to prove that $\varphi$ has a local linking at 0 with respect to $X^{\alpha}$. From Theorem 2.7, $X^{\alpha}$ has a countable orthogonal basis $\left\{w_{i}\right\}$. Set $Y_{k}=\operatorname{span}\left\{w_{1}, w_{2}, \ldots w_{k}\right\}$ and $Z_{k}=Y_{k}^{\perp}$. Then $X^{\alpha}=Y_{k} \oplus Z_{k}$. 
We divide the proof into the following two steps.

Step 1. Take $u \in Y_{k}$. Since $Y_{k}$ is finite dimensional, we have that for given $r_{0}$, there exists $0<\rho<1$ small such that $u \in Y_{k},\|u\|_{X^{\alpha}} \leq \rho \rightarrow|u(t)|<r_{0}, t \in \mathbb{R}$. For $0<r<r_{0}$, let $\Omega_{1}=\{t \in \mathbb{R}:|u(t)|<r\}, \Omega_{2}=$ $\left\{t \in \mathbb{R}: r \leq|u(t)| \leq r_{0}\right\}$ and $\Omega_{3}=\left\{t \in \mathbb{R}:|u(t)|>r_{0}\right\}$. Then $\mathbb{R}=\bigcup_{i=1}^{3} \Omega_{i}$. For the sake of simplicity, let $H(t, u(t))=F(t, u(t))-m_{1}|u(t)|^{\gamma}$. Hence, we can get

$$
\begin{aligned}
-\int_{\mathbb{R}} F(t, u(t)) d t & =-\int_{\mathbb{R}} m_{1}|u(t)|^{\gamma} d t-\int_{\mathbb{R}} H(t, u(t)) d t \\
& =-\int_{\mathbb{R}} m_{1}|u(t)|^{\gamma} d t-\left(\int_{\Omega_{1}}+\int_{\Omega_{2}}+\int_{\Omega_{3}}\right) H(t, u(t)) d t \\
& \leq-\int_{\mathbb{R}} m_{1}|u(t)|^{\gamma} d t-\int_{\Omega_{1}} H(t, u(t)) d t \\
& =-m_{1}\|u\|_{\gamma}^{\gamma}-\int_{\Omega_{1}} H(t, u(t)) d t .
\end{aligned}
$$

by (2.7), (3.14) and (f5), we have

$$
\begin{aligned}
\varphi(u)= & \frac{a}{2} \int_{\mathbb{R}}\left(\left|{ }_{-\infty} D_{t}^{\alpha} u(t)\right|^{2}+b(t)|u(t)|^{2}\right) d t+\frac{\lambda}{2(\tau+1)}\left(\int_{\mathbb{R}}\left(\left|{ }_{-\infty} D_{t}^{\alpha} u(t)\right|^{2}+b(t)|u(t)|^{2}\right) d t\right)^{\tau+1} \\
& -\int_{\mathbb{R}} F(t, u(t)) d t \\
= & \frac{a}{2}\|u\|_{X^{\alpha}}^{2}+\frac{\lambda}{2(\tau+1)}\|u\|_{X^{\alpha}}^{2(\tau+1)}-\int_{\mathbb{R}} F(t, u(t)) d t \\
\leq & \frac{a}{2}\|u\|_{X^{\alpha}}^{2}+\frac{\lambda}{2(\tau+1)}\|u\|_{X^{\alpha}}^{2(\tau+1)}-m_{1}\|u\|_{\gamma}^{\gamma}-\int_{\Omega_{1}} H(t, u(t)) d t
\end{aligned}
$$

for all $u \in Y_{k}$. Since the norms on $Y_{k}$ are equivalent to each other, $\|u\|_{\gamma}$ is equivalent to $\|u\|_{X^{\alpha}}$, we find that $\int_{\Omega_{1}} H(t, u(t)) d t \rightarrow 0$ as $r \rightarrow 0$. Since $1<\gamma<2$, we have $\varphi(u) \leq 0$, for all $u \in Y_{k}$ with $\|u\|_{X^{\alpha}} \leq \rho$.

Step 2. Take $u \in Z_{k}$. Using Remark 2.6, we have that for given $r_{0}$, there exists $0<\rho<1$ small such that

$$
u \in Z_{k},\|u\|_{X^{\alpha}} \leq \rho \rightarrow|u(t)|<r_{0}, t \in \mathbb{R} .
$$

Then, (2.6), (2.7) and (f5) imply that

$$
\begin{aligned}
\varphi(u)= & \frac{a}{2} \int_{\mathbb{R}}\left(\left.\left.\right|_{-\infty} D_{t}^{\alpha} u(t)\right|^{2}+b(t)|u(t)|^{2}\right) d t+\frac{\lambda}{2(\tau+1)}\left(\int_{\mathbb{R}}\left(\left|{ }_{-\infty} D_{t}^{\alpha} u(t)\right|^{2}+b(t)|u(t)|^{2}\right) d t\right)^{\tau+1} \\
& -\int_{\mathbb{R}} F(t, u(t)) d t \\
= & \frac{a}{2}\|u\|_{X^{\alpha}}^{2}+\frac{\lambda}{2(\tau+1)}\|u\|_{X^{\alpha}}^{2(\tau+1)}-\int_{\mathbb{R}} F(t, u(t)) d t \\
\geq & \frac{a}{2}\|u\|_{X^{\alpha}}^{2}-\int_{\mathbb{R}} F(t, u(t)) d t \\
\geq & \frac{a}{2}\|u\|_{X^{\alpha}}^{2}-m_{2} \int_{\mathbb{R}}|u(t)|^{2} d t \\
> & \frac{a}{2}\|u\|_{X^{\alpha}}^{2}-\frac{a}{2}\|u\|_{X^{\alpha}}^{2}=0 .
\end{aligned}
$$

Therefore, $\varphi(u)>0$, for all $u \in Z_{k}$ with $\|u\|_{X^{\alpha}} \leq \rho$. Consequently, by Theorem 2.13, 0 is a homological nontrivial critical point of $\varphi$. This completes the proof. 
Now, we need the following lemma to prove of Theorem 1.1.

Lemma 3.3. Suppose that (B), $(f 1)$ and $(f 2)$ hold, and $\left\{u_{n}\right\} \subset X^{\alpha}$ is a bounded sequence with $\varphi^{\prime}\left(u_{n}\right) \rightarrow 0$. Then $\left\{u_{n}\right\}$ has a convergent subsequence.

Proof. Note that $\left\{u_{n}\right\} \subset X^{\alpha}$ is bounded and $X^{\alpha}$ is a reflexive Banach space. Passing to a subsequence (for simplicity denoted gain by $\left\{u_{n}\right\}$ ) if necessary, by Remark 2.6, we may assume that

$$
\begin{cases}u_{n} \rightarrow u, & \text { weakly in } X^{\alpha}, \\ u_{n} \rightarrow u, & \text { strongly in } L^{p}(\mathbb{R}), \quad 2 \leq p<\infty .\end{cases}
$$

Note that

$$
\begin{aligned}
\left\langle\varphi^{\prime}\left(u_{n}\right)-\varphi^{\prime}(u), u_{n}-u\right\rangle= & a \int_{\mathbb{R}}\left(\left|{ }_{-\infty} D_{t}^{\alpha}\left(u_{n}-u\right)\right|^{2}+b(t)\left|u_{n}-u\right|^{2}\right) d t \\
& -\lambda\left[\left(\int_{\mathbb{R}}\left(\left.\left.\right|_{-\infty} D_{t}^{\alpha} u\right|^{2}+b(t)|u|^{2}\right) d t\right)^{\tau}\right. \\
& \cdot \int_{\mathbb{R}}\left({ }_{-\infty} D_{t}^{\alpha} u{ }_{-\infty} D_{t}^{\alpha}\left(u_{n}-u\right)+b(t) u\left(u_{n}-u\right)\right) d t \\
& -\left(\int_{\mathbb{R}}\left(\left|{ }_{-\infty} D_{t}^{\alpha} u_{n}\right|^{2}+b(t)\left|u_{n}\right|^{2}\right) d t\right)^{\tau} \\
& \left.\cdot \int_{\mathbb{R}}\left({ }_{-\infty} D_{t}^{\alpha} u_{n-\infty} D_{t}^{\alpha}\left(u_{n}-u\right)+b(t) u_{n}\left(u_{n}-u\right)\right) d t\right] \\
& -\int_{\mathbb{R}}\left(f\left(t, u_{n}\right)-f(t, u)\right) \cdot\left(u_{n}-u\right) d t \\
= & a\left\|u_{n}-u\right\|_{X^{\alpha}}^{2} \\
& -\lambda\left[\|u\|_{X^{\alpha}}^{2 \tau} \cdot \int_{\mathbb{R}}\left({ }_{-\infty} D_{t}^{\alpha} u{ }_{-\infty} D_{t}^{\alpha}\left(u_{n}-u\right)+b(t) u\left(u_{n}-u\right)\right) d t\right. \\
& \left.-\left\|u_{n}\right\|_{X^{\alpha}}^{2 \tau} \cdot \int_{\mathbb{R}}\left({ }_{-\infty} D_{t}^{\alpha} u_{n-\infty} D_{t}^{\alpha}\left(u_{n}-u\right)+b(t) u_{n}\left(u_{n}-u\right)\right) d t\right] \\
& -\int_{\mathbb{R}}\left(f\left(t, u_{n}\right)-f(t, u)\right) \cdot\left(u_{n}-u\right) d t .
\end{aligned}
$$

It follows that

$$
\begin{aligned}
a\left\|u_{n}-u\right\|_{X^{\alpha}}^{2}= & \left\langle\varphi^{\prime}\left(u_{n}\right)-\varphi^{\prime}(u), u_{n}-u\right\rangle \\
& +\lambda\left[\|u\|_{X^{\alpha}}^{2 \tau} \cdot \int_{\mathbb{R}}\left({ }_{-\infty} D_{t}^{\alpha} u_{-\infty} D_{t}^{\alpha}\left(u_{n}-u\right)+b(t) u\left(u_{n}-u\right)\right) d t\right. \\
& \left.-\left\|u_{n}\right\|_{X^{\alpha}}^{2 \tau} \cdot \int_{\mathbb{R}}\left({ }_{-\infty} D_{t}^{\alpha} u_{n-\infty} D_{t}^{\alpha}\left(u_{n}-u\right)+b(t) u_{n}\left(u_{n}-u\right)\right) d t\right] \\
& +\int_{\mathbb{R}}\left(f\left(t, u_{n}\right)-f(t, u)\right) \cdot\left(u_{n}-u\right) d t
\end{aligned}
$$

Set $\widetilde{X}^{\alpha}=\left\{u \in L^{2}(\mathbb{R}):{ }_{-\infty} D_{t}^{\alpha} u \in L^{2}(\mathbb{R})\right\}$ with the norm $\|u\|_{\widetilde{X}^{\alpha}}=\left(\int_{\mathbb{R}}\left(\left|{ }_{-\infty} D_{t}^{\alpha} u(t)\right|^{2}+|u(t)|^{2}\right) d t\right)^{\frac{1}{2}}$. By (B1), we have

$$
\min \left\{1, b_{1}\right\} \cdot\|u\|_{\widetilde{X}^{\alpha}} \leq\left(\int_{\mathbb{R}}\left(\left|{ }_{-\infty} D_{t}^{\alpha} u(t)\right|^{2}+b(t)|u(t)|^{2}\right) d t\right)^{\frac{1}{2}}=\|u\|_{X^{\alpha}} .
$$


Then the imbedding $X^{\alpha} \hookrightarrow \widetilde{X}^{\alpha}$ is continuous. By the continuity of the imbedding $X^{\alpha} \hookrightarrow \widetilde{X}^{\alpha}$, one has $u_{n} \rightarrow u$ in $\widetilde{X}^{\alpha}$. Hence,

$$
\int_{\mathbb{R}}\left|{ }_{-\infty} D_{t}^{\alpha}\left(u_{n}-u\right)\right|^{2} d t+\int_{\mathbb{R}}\left|u_{n}-u\right|^{2} d t \rightarrow 0 \text {, as } n \rightarrow \infty .
$$

By the boundedness of $\left\{u_{n}\right\}$ in $X^{\alpha}$, we can get

$$
\begin{aligned}
& \lambda\left[\|u\|_{X^{\alpha}}^{2 \tau} \cdot \int_{\mathbb{R}}\left({ }_{-\infty} D_{t}^{\alpha} u_{-\infty} D_{t}^{\alpha}\left(u_{n}-u\right)+b(t) u\left(u_{n}-u\right)\right) d t\right. \\
& \left.-\left\|u_{n}\right\|_{X^{\alpha}}^{2 \tau} \cdot \int_{\mathbb{R}}\left({ }_{-\infty} D_{t}^{\alpha} u_{n-\infty} D_{t}^{\alpha}\left(u_{n}-u\right)+b(t) u_{n}\left(u_{n}-u\right)\right) d t\right] \rightarrow 0, \text { as } n \rightarrow \infty .
\end{aligned}
$$

Moreover, by (2.9), for any $\varepsilon>0$, one has

$$
\begin{aligned}
\left|\int_{\mathbb{R}}\left(f\left(t, u_{n}\right)-f(t, u)\right) \cdot\left(u_{n}-u\right) d t\right| \leq & \int_{\mathbb{R}}\left(\varepsilon\left(\left|u_{n}\right|+|u|\right)+c(\varepsilon)\left(\left|u_{n}\right|^{p-1}+|u|^{p-1}\right)\right) \cdot\left|u_{n}-u\right| d t \\
\leq & \varepsilon\left(\left\|u_{n}\right\|_{2}+\|u\|_{2}\right)\left\|u_{n}-u\right\|_{2}+c(\varepsilon)\left\|u_{n}-u\right\|_{p} \\
& \cdot\left(\left\|u_{n}\right\|_{p}^{p-1}+\|u\|_{p}^{p-1}\right) \\
\leq & \varepsilon c_{2}\left\|u_{n}-u\right\|_{2}+c_{3} c(\varepsilon)\left\|u_{n}-u\right\|_{p},
\end{aligned}
$$

where $c_{2}$ and $c_{3}$ are independent of $\varepsilon$ and $n$. In view of (3.15), one can get

$$
\int_{\mathbb{R}}\left(f\left(t, u_{n}\right)-f(t, u)\right) \cdot\left(u_{n}-u\right) d t \rightarrow 0, \text { as } n \rightarrow \infty .
$$

Note that $\varphi^{\prime}\left(u_{n}\right) \rightarrow 0$. Using (3.16), (3.17) and (3.18), we have that $\left\|u_{n}-u\right\|_{X^{\alpha}} \rightarrow 0$. This completes the proof.

Proof of Theorem 1.1. We shall apply Theorem 2.12 to $\varphi$. We know that $X^{\alpha}$ is a Banach space and $\varphi \in C^{1}\left(X^{\alpha}, \mathbb{R}\right)$ (see Lemma 2.16).

By (f3), similar to Lemma 2.3 in [20], there exists a constant $\eta>2(\tau+1)$ such that $\eta<\mu$, and

$$
\lim _{|u| \rightarrow \infty} \frac{F(t, u)}{|u|^{\eta}}=+\infty, \text { uniformly in } t \in \mathbb{R}
$$

Consequently,

$$
\lim _{|u| \rightarrow \infty} \frac{F(t, u)}{|u|^{2(\tau+1)}}=+\infty, \text { uniformly in } t \in \mathbb{R} .
$$

Hence, for any $0<\varepsilon<\frac{a}{C_{2}^{2}}\left(C_{2}\right.$ appears in (2.4)), by (2.4), (2.7) and (2.10), for small $\rho>0$,

$$
\begin{aligned}
\varphi(u)= & \frac{a}{2} \int_{\mathbb{R}}\left(\left|{ }_{-\infty} D_{t}^{\alpha} u(t)\right|^{2}+b(t)|u(t)|^{2}\right) d t+\frac{\lambda}{2(\tau+1)}\left(\int_{\mathbb{R}}\left(\left|-_{-\infty} D_{t}^{\alpha} u(t)\right|^{2}+b(t)|u(t)|^{2}\right) d t\right)^{\tau+1} \\
& -\int_{\mathbb{R}} F(t, u(t)) d t
\end{aligned}
$$




$$
\begin{aligned}
& =\frac{a}{2}\|u\|_{X^{\alpha}}^{2}+\frac{\lambda}{2(\tau+1)}\|u\|_{X^{\alpha}}^{2(\tau+1)}-\int_{\mathbb{R}} F(t, u(t)) d t \\
& \geq \frac{a}{2}\|u\|_{X^{\alpha}}^{2}-\int_{\mathbb{R}} F(t, u(t)) d t \\
& \geq \frac{a}{2}\|u\|_{X^{\alpha}}^{2}-\frac{\varepsilon}{2}\|u\|_{2}^{2}-\frac{c(\varepsilon)}{p}\|u\|_{p}^{p} \\
& \geq \frac{a}{2}\|u\|_{X^{\alpha}}^{2}-\frac{\varepsilon}{2} c_{2}^{2}\|u\|_{X^{\alpha}}^{2}-\frac{c(\varepsilon)}{p} C_{p}^{p}\|u\|_{X^{\alpha}}^{p} \\
& =\frac{1}{2}\left(a-C_{2}^{2} \varepsilon\right)\|u\|_{X^{\alpha}}^{2}-\frac{c(\varepsilon)}{p} C_{p}^{p}\|u\|_{X^{\alpha}}^{p} \\
& \geq \frac{1}{4}\left(a-C_{2}^{2} \varepsilon\right)\|u\|_{X^{\alpha}}^{2}
\end{aligned}
$$

for all $u \in \bar{B}_{\rho}$, where $B_{\rho}=\left\{u \in X^{\alpha}:\|u\|_{X^{\alpha}}<\rho\right\}$. Therefore,

$$
\left.\varphi\right|_{\partial B_{\rho}} \geq \frac{1}{4}\left(a-C_{2}^{2} \varepsilon\right) \rho^{2}:=\beta>0
$$

Therefore,

$$
\left.\varphi\right|_{\partial B_{\rho} \cap Z_{k}} \geq \beta>0
$$

Moreover, for any finite dimensional subspace $\widehat{X}^{\alpha} \subset X^{\alpha}$, there is a positive integer number $m$ such that $\widehat{X}^{\alpha} \subset Y_{m}$. Since all norms are equivalent in a finite dimensional space, there is a constant $\gamma>0$ such that

$$
\|u\|_{\eta} \geq \gamma\|u\|_{X^{\alpha}}, \forall u \in Y_{m}
$$

Take $0 \neq u_{0} \in Y_{m}$. In view of (f1) and (3.20), we can get, for any $M_{\eta}>\frac{\lambda\left\|u_{0}\right\|_{X \alpha}^{2(\tau+1)}}{(\tau+1)\left\|u_{0}\right\|_{X}^{\eta} \gamma^{\eta}}$, there exists a constant $C\left(M_{\eta}\right)$ such that

$$
F\left(t, u_{0}\right) \geq M_{\eta}\left|u_{0}\right|^{2(\tau+1)}-C\left(M_{\eta}\right)\left|u_{0}\right|^{2} .
$$

So, by (2.4) and (3.23), we have

$$
\begin{aligned}
\varphi\left(x u_{0}\right) & =\frac{a x^{2}}{2} \int_{\mathbb{R}}\left(\left.\left.\right|_{-\infty} D_{t}^{\alpha} u_{0}(t)\right|^{2}+b(t)\left|u_{0}(t)\right|^{2}\right) d t \\
& +\frac{\lambda x^{2(\tau+1)}}{2(\tau+1)}\left(\int_{\mathbb{R}}\left(\left.||_{-\infty} D_{t}^{\alpha} u_{0}(t)\right|^{2}+b(t)\left|u_{0}(t)\right|^{2}\right) d t\right)^{\tau+1}-\int_{\mathbb{R}} F\left(t, u_{0}(t)\right) d t \\
& \leq \frac{a x^{2}}{2}\left\|u_{0}\right\|_{X^{\alpha}}^{2}+\frac{\lambda x^{2(\tau+1)}}{2(\tau+1)}\left\|u_{0}\right\|_{X^{\alpha}}^{2(\tau+1)}-M_{\eta} x^{2(\tau+1)}\left\|u_{0}\right\|_{2(\tau+1)}^{2(\tau+1)}+C\left(M_{\eta}\right) x^{2}\|u\|_{2}^{2} \\
& \leq \frac{a x^{2}}{2}\left\|u_{0}\right\|_{X^{\alpha}}^{2}+\frac{\lambda x^{2(\tau+1)}}{2(\tau+1)}\left\|u_{0}\right\|_{X^{\alpha}}^{2(\tau+1)}-M_{\eta} x^{2(\tau+1)} \gamma^{2(\tau+1)}\left\|u_{0}\right\|_{X^{\alpha}}^{2(\tau+1)}+C\left(M_{\eta}\right) C_{2}^{2} x^{2}\|u\|_{X^{\alpha}}^{2} \\
& \leq \frac{a x^{2}}{2}\left\|u_{0}\right\|_{X^{\alpha}}^{2}+x^{2(\tau+1)}\left(\frac{\lambda}{2(\tau+1)}-M_{\eta} \gamma^{2(\tau+1)}\right)\left\|u_{0}\right\|_{X^{\alpha}}^{2(\tau+1)}+C\left(M_{\eta}\right) C_{2}^{2} x^{2}\|u\|_{X^{\alpha}}^{2}
\end{aligned}
$$

for all $u \in Y_{m}$. Consequently, there is a large $r_{1}>0$ such that $\varphi<0$ on $\widetilde{X}^{\alpha} \backslash B_{r_{1}}$. Consequently, there is a point $e \in X^{\alpha}$ with $\|e\|_{X^{\alpha}}>\rho$ such that $\varphi(e)<0$. Moreover, we know that $\varphi(0)=0$. Consequently, by 
(3.22), we have

$$
\left.\varphi\right|_{\partial B_{\rho} \cap Z_{k}}>0=\max (\varphi(0), \varphi(e)) .
$$

Now, we prove that $\varphi$ satisfies the (PS)-condition. Indeed, if we have a sequence $\left\{u_{n}\right\} \subset X^{\alpha}$ such that $\left\{\varphi\left(u_{n}\right)\right\}$ is bounded and $\varphi^{\prime}\left(u_{n}\right) \rightarrow 0$, we need to prove that $\left\{u_{n}\right\}$ possesses a convergent subsequence. By Lemma 3.3, we know that it is sufficient to prove that $\left\{u_{n}\right\}$ is bounded in $X^{\alpha}$. If $\left\{u_{n}\right\}$ is unbounded in $X^{\alpha}$, we can assume that $\left\|u_{n}\right\|_{X^{\alpha}} \rightarrow+\infty$. Setting $v_{n}=\frac{u_{n}}{\left\|u_{n}\right\|_{X^{\alpha}}}$, we have $\left\|v_{n}\right\|_{X^{\alpha}}=1$ and $\left\|v_{n}\right\|_{p} \leq c_{p}\left\|v_{n}\right\|_{X^{\alpha}}=c_{p}$ for each $2 \leq p<\infty$. Note that

$$
\frac{\left\langle\varphi^{\prime}\left(u_{n}\right), u_{n}\right\rangle}{\left\|u_{n}\right\|_{X^{\alpha}}^{\eta}}=a \frac{\left\|u_{n}\right\|_{X^{\alpha}}^{2}}{\left\|u_{n}\right\|_{X^{\alpha}}^{\eta}}+\lambda \frac{\left\|u_{n}\right\|_{X^{\alpha}}^{2(\tau+1)}}{\left\|u_{n}\right\|_{X^{\alpha}}^{\eta}}-\frac{\int_{\mathbb{R}} f\left(t, u_{n}\right) u_{n} d t}{\left\|u_{n}\right\|_{X^{\alpha}}^{\eta}} .
$$

Letting $n \rightarrow+\infty$, we obtain from $\eta>2(\tau+1)$ that

$$
\lim _{n \rightarrow+\infty} \int_{\mathbb{R}} \frac{f\left(t, u_{n}\right) u_{n}}{\left\|u_{n}\right\|_{X^{\alpha}}^{\eta}} d t=0 .
$$

Since $X^{\alpha}$ is a reflexive Banach space and $\left\|v_{n}\right\|_{X^{\alpha}}=1$, passing to a subsequence, by (2.4), there is a point $v \in X^{\alpha}$ such that $\left\{v_{n}\right\}$ converges weakly to $v$ in $X^{\alpha}$, that it converges to $v$ in $L^{p}(\mathbb{R})$ and that $v_{n}(t) \rightarrow v(t)$ for a.e. $t \in \mathbb{R}$, where $2 \leq p<\infty$. Set $\Omega=\{t \in \mathbb{R}: v(t) \neq 0\}$. If meas $(\Omega)>0$, then $\left|u_{n}(t)\right| \rightarrow+\infty$ for a.e. $t \in \Omega$. By (f1) and (f2) there are positive constants $b_{2}$ and $b_{3}$ such that

$$
f(t, u) u \geq b_{2}|u|^{\eta}-b_{3}|u|^{2}, \quad \forall(t, u) \in \mathbb{R} \times \mathbb{R} .
$$

Hence by (3.24), we have

$$
\int_{\mathbb{R}} \frac{f\left(t, u_{n}\right) u_{n}}{\left\|u_{n}\right\|_{X^{\alpha}}^{\eta}} d t \geq b_{2}\left\|v_{n}\right\|_{\eta}^{\eta}-b_{3} \frac{\left\|v_{n}\right\|_{2}^{2}}{\left\|u_{n}\right\|_{X^{\alpha}}^{\eta-2}}
$$

Consequently,

$$
0=\lim _{n \rightarrow+\infty} \int_{\mathbb{R}} \frac{f\left(t, u_{n}\right) u_{n}}{\left\|u_{n}\right\|_{X^{\alpha}}^{\eta}} d t \geq b_{2}\|v\|_{\eta}^{\eta}=\int_{\Omega}|v|^{\eta} d t>0
$$

This is a contradiction. Hence meas $(\Omega)=0$. Therefore, $v(t)=0$ for a.e. $t \in \mathbb{R}$. Moreover, by (f1)-(f3), since $\mu>2(\tau+1)>2$ and $p>2$, there is a constant $b_{4}>0$ such that

$$
\frac{1}{\mu} f(t, u) u-F(t, u) \geq-\varepsilon\left(\frac{1}{2}+\frac{1}{\mu}\right)|u|^{2}-c(\varepsilon)\left(\frac{1}{\mu}+\frac{1}{p}\right)|u|^{p} \geq-b_{4}|u|^{2}
$$

for all $(t, u) \in \mathbb{R} \times \mathbb{R}$. Consequently, by (3.25), we have

$$
\begin{aligned}
\frac{1}{\left\|u_{n}\right\|_{X^{\alpha}}^{2}}\left[\varphi\left(u_{n}\right)-\frac{1}{\mu}\left\langle\varphi^{\prime}\left(u_{n}\right), u_{n}\right\rangle\right] & \geq\left(\frac{1}{2}-\frac{1}{\mu}\right) a+\frac{\int_{\mathbb{R}}\left(\frac{1}{\mu} f\left(t, u_{n}\right) u_{n}-F\left(t, u_{n}\right)\right) d t}{\left\|u_{n}\right\|_{X^{\alpha}}^{2}} \\
& \geq\left(\frac{1}{2}-\frac{1}{\mu}\right) a-b_{4} \frac{1}{\left\|u_{n}\right\|_{X^{\alpha}}^{2}}\left\|u_{n}\right\|_{2}^{2} .
\end{aligned}
$$

Letting $n \rightarrow+\infty$, since $\left\|u_{n}\right\|_{X^{\alpha}} \rightarrow+\infty$, we obtain $0 \geq\left(\frac{1}{2}-\frac{1}{\mu}\right) a$. Since $a>0, \lambda>0, \tau \geq 0$ and $\mu>$ $2(\tau+1)>2$, this implies $0 \geq\left(\frac{1}{2}-\frac{1}{\mu}\right) a$, that it is a contradiction. So, $\left\{u_{n}\right\}$ is bounded in $X^{\alpha}$. Therefore, by Lemma 3.3, $\varphi$ satisfies the (PS)-condition. This completes the proof.

Proof of Theorem 1.2. We apply Theorem 2.14 to $\varphi$. By Lemma 2.16, $\varphi \in C^{1}\left(X^{\alpha}, \mathbb{R}\right)$. Lemma 3.1 shows that $\varphi$ satisfies the (PS)-condition and is bounded from below. By Lemma 3.2, there exists $k_{0} \in \mathbb{N}$ 
such that $C_{k_{0}}(\varphi, 0) \varsubsetneqq 0$. Consequently, by Theorem 2.13, 0 is a homological nontrivial critical point of $\varphi$ but not a minimizer. By Theorem 2.14, we get that problem (1.1) has at least two nontrivial solutions. The proof is complete.

Proof of Theorem 1.3. We apply Theorem 2.15 to $\varphi$. By (f6) and (2.7), one can easily check that functional $\varphi$ is even and satisfies $\varphi(0)=0$. By Lemma 2.16, $\varphi \in C^{1}\left(X^{\alpha}, \mathbb{R}\right)$. Lemma 3.1 shows that $\varphi$ satisfies the (PS)-condition and is bounded from below. For $\rho>0$, let $K=S_{\rho}=\left\{u \in Y_{k}:\|u\|_{X^{\alpha}}=\rho\right\}$. So, as shown in the proof of Lemma 3.2,

$$
\varphi(u) \leq \frac{a}{2}\|u\|_{X^{\alpha}}^{2}+\frac{\lambda}{2(\tau+1)}\|u\|_{X^{\alpha}}^{2(\tau+1)}-m_{1}\|u\|_{\eta}^{\eta}-\int_{\Omega_{1}} H(t, u(t)) d t
$$

for all $u \in Y_{k}$. Note that the norms on $Y_{k}$ are equivalent to each other, $\|u\|_{\gamma}$ is equivalent to $\|u\|_{X^{\alpha}}$ and $\int_{\Omega_{1}} H(t, u(t)) d t \rightarrow 0$ as $r \rightarrow 0$. Since $\eta>2(\tau+1)$, we have $\varphi(u) \leq 0$, for all $u \in Y_{k}$ with $\|u\|_{X^{\alpha}} \leq \rho$. If $\rho>0$ is small enough, one has $\sup _{K} \varphi(u)<0$. By the definition of $Y_{k}$, we have $\operatorname{dim}\left(Y_{k}\right)=k \operatorname{Using}$ Theorem 2.15, we have that $\varphi$ has at least $k$ distinct pairs of critical points. Therefore, problem (1.1) has at least $k$ distinct pairs of solutions. The proof is complete.

\section{Acknowledgements}

The authors would like to thank the referees for their suggestions and helpful comments which improved the presentation of the original manuscript.

\section{REFERENCES}

[1] A.A. Kilbas, H.M. Srivastava, J.J. Trujillo, Theory and Applications of Fractional Differential Equations, in: NorthHolland Mathematics Studies, vol. 204, Elsevier Science B.V., Amsterdam, (2006).

[2] O. Agrawal, J.T. Machado, J. Sabatier, Fractional Derivatives and their Application: Nonlinear Dynamics, SpringerVerlag, Berlin, (2004).

[3] R. Hilfer, Applications of Fractional Calculus in Physics, World Scientific, Singapore, (2000).

[4] K.S. Miller, B. Ross, An Introduction to the Fractional Calculus and Fractional Differential Equations, Wiley, New York, (1993).

[5] I. Podlubny, Fractional Differential Equations, Academic Press, New York, (1999).

[6] J. Sabatier, O. Agrawal, J.T. Machado, Advances in Fractional Calculus, Theoretical Developments and Applications in Physics and Engineering. Springer-Verlag, Berlin, (2007).

[7] G. Zaslavsky, Hamiltonian Chaos and Fractional Dynamics, Oxford University Press, (2005).

[8] C. Torres, Existence of solution for a class of fractional Hamiltonian systems, Electron. J. Differential Equations 2013 (2013), Article ID 259.

[9] A. Méndez, C. Torres, Multiplicity of solutions for fractional Hamiltonian systems with Liouville-Weyl fractional derivative, Frac. Calc. Appl. Anal. 18 (2016), 875-890.

[10] C. Torres, Tempered fractional differential equation: variational approach, Math. Meth. Appl. Sci. 40 (2017), 4962-4973

[11] N. Nyamoradi, Y. Zhou, Existence of solutions for a Kirchhoff type fractional differential equations via minimal principle and Morse theorys, Topolog. Meth. Appl. Anal. 76 (2015), 617-630.

[12] J. Xu, D. O'Regan, K. Zhang, Multiple solutions for a class of fractional Hamiltonian systems, Fract. Calc. Appl. Anal. 18 (2015) 48-63.

[13] Z. Zhang, R. Yuan, Variational approach to solutions for a class of fractional Hamiltonian systems, Math. Methods Appl. Sci. 37 (2014) 1873-1883.

[14] C. Chen, Q. Zhu, Existence of positive solutions to p-Kirchhoff-type problem without compactness conditions, Appl. Math. Lett. 28 (2014), 82-87. 
[15] P. Pucci, M.Q. Xiang, B.L. Zhang, Multiple solutions for nonhomogeneous Schrödinger-Kirchhoff type equations involving the fractional $p$-Laplacian in $\mathbb{R}^{N}$, Calc. Var. Partial Differential Equations 54 (2015), 2785-2806.

[16] N.T. Chung, Multiple solutions for a $p(x)$-Kirchhoff-type equation with sign-changing nonlinearities, Complex Var. Elliptic Equ. 58 (2013) 1637-1646.

[17] G. Dai, R. Hao, Existence of solutions for a $p(x)$-Kirchhoff-type equation, J. Math. Anal. Appl. 359 (2009) $275-284$.

[18] D. Liu, On a $p$-Kirchhoff equation via fountain theorem and dual fountain theorem, Nonlinear Anal. 72 (2010), $302-308$.

[19] J. Nie, Existence and multiplicity of nontrivial solutions for a class of Schrödinger-Kirchhoff-type equations, J. Math. Anal. Appl. 417 (2014), 65-79.

[20] Y. Guo, J. Nie, Existence and multiplicity of nontrivial solutions for p-Laplacian Schrödinger-Kirchhoff-type equations, J. Math. Anal. Appl. 428 (2015), 1054-1069.

[21] M.Q. Xiang, B.L. Zhang, Degenerate Kirchhoff problems involving the fractional $p$-Laplacian without the (AR) condition, Complex Var. Elliptic Equ. 60 (2015), 1277-1287.

[22] V. Ervin, J. Roop, Variational formulation for the stationary fractional advection dispersion equation, Numer. Methods Partial Differential Equations 22 (2006) 58-76.

[23] F. Jiao, Y. Zhou, Existence of solutions for a class of fractional boundary value problems via critical point theory, Comput. Math. Appl. 62 (2011) 1181-1199.

[24] J. Mawhin, M. Willem, Critical Point Theory and Hamiltonian Systems, Appl. Math. Sci. 74, Springer, New York, (1989).

[25] J.Q. Liu, J. Su, Remarks on multiple nontrivial solutions for quasilinear resonant problems, J. Math. Anal. Appl. 258 (2001), 209-222.

[26] P.H. Rabinowitz, Minimax Method in Critical Point Theory with Applications to Differential Equations, CBMS Regional Conference Series in Mathematics, 65. Published for the Conference Board of the Mathematical Sciences, Washington, DC; by the American Mathematical Society, Providence, RI, 1986.

[27] M. Willem, Minimax Theorem, Boston, MA: Birkhäuser, (1996). 